\title{
Towards the development of automated fed-batch cell culture processes at microscalle
}

Vincent Wiegmann', Maria Giaka', Cristina Bernal Martinez² \& Frank Baganz*,1

\section{ABSTRACT}

Aim: To investigate the impact of various feeding strategies on the growth and productivity of a GS-CHO cell line. Methods: Feed additions were conducted at fixed volumes or linked to a marker such as cell growth or metabolismand added as bolus ornear-continuously using the automated feeding module of the micro-Matrix (Applikon). Results: The selected feeding regimens supported maximum viable cell densities of up to $1.9 \times 10^{7} \mathrm{cells} \mathrm{ml}^{-1}$ and final titers of up to $1.13 \mathrm{gl}^{-1}$. Differences in growth and titer between feeding strategies were insignificant, with the exception of one feeding strategy. Conclusion: As the more complex feeding strategies did not create an advantage, the selection of a simple feeding strategy such as bolus or continuous addition of feed medium is preferred.

\section{METHOD SUMMARY}

Fed-batch is an easy and popular way to intensify cell cultivations. The time of addition and the volume that is added can be decisive in achieving optimal product titers. A micro-bioreactor has been used to investigate the effect of several feeding strategies on the growth and production kinetics of a GS-CHO cell line.

\section{KEYWORDS}

automation $\cdot$ downscale $\cdot$ fed-batch - GS-CHO cells • miniature shaken bioreactor

'The Advanced Centre for Biochemical Engineering, Department of Biochemical Engineering, University College London, Gower Street, London WC1E 6BT, UK; ${ }^{2}$ Applikon-Biotechnology BV. Heertjeslaan 2, 2629JG, Delft, The Netherlands; *Author for correspondence:f.baganz@ucl.ac.uk

BioTechniques 67: 238-241 (November 2019) $10.2144 /$ btn-2019-0063
In recent years, microbioreactors have transformed early-stage process development by providing controlled culture environments at a high degree of parallelization and short turnover times [1-5]. Applikon's (The Netherlands) micro-Matrix is a platform that holds 24 microbioreactors on a deep-square well format and offers full control of $\mathrm{pH}$ and dissolved oxygen in each well [6]. Additionally, the micro-Matrix is equipped with an automated feeding module, which can be used for near-continuous additions of liquids such as base or feed medium. The addition of feed medium is a popular way to enhance the cell growth and productivity of biopharmaceutical production processes. The high productivity compared with cultivations in batch, as well as the ease of scale translation and operability compared with perfusion, often render fed-batch as the preferred mode of operation [7-9]. The inflowing nutrient solution aims to replenish critical media components, and thereby supports further cell growth and delays the onset of cell death. The culture volume increases over time through the addition of feed medium, whereby the addition can follow various protocols. The influx of feed medium can either be continuous or intermittent, or it can be linked to certain events during the cultivation, such as the glucose concentration dropping below a specified level. At the end of the run, the reactor content is either harvested entirely or some of the cell broth remains in the vessel, serving as inoculum for a subsequent run (repeated fed-batch) [10]. Early feeding strategies tested the addition of the basal medium enriched with glucose [11] and subsequent approaches began to more closely inspect other media components, resulting in more tailored feed media [12-14]. More recently, dynamic strategies that involve media blending or spent media analysis further improved fed-batch processes [15]. In order to avoid the inhibitory effects that some medium components exert at increased levels, the high concentrations in the fortified growth media often make a gradual addition preferable [11]. As glucose is not necessarily the limiting nutrient in every process, a comprehensive metabolic footprint of the cell line in use is vital for designing a tailored feed medium. Further growth is usually inhibited through the accumulation of one or more metabolic waste products.

Besides simple bolus additions of feed medium at regular intervals $[16,17]$, various alternative feeding strategies have been reported in the literature. Additions of feed medium have, for instance, been coupled to glucose or glutamine consumption $[18,19]$ to tailor the feeding regimen to the metabolic needs of the cell population. The cell concentration has also successfully been used as a basis for feed additions [20]. A recent study describes the use of in silico models in combination with design of experiments (DoE) to accelerate the optimization of fed-batch strategies [21]. Furthermore, feed medium can be supplied in a continuous fashion rather than in bolus shots to avoid spikes in the availability of nutrients [22].

The systematic investigation of feeding strategies at small scale is often limited to simple bolus additions of feed medium, as microtiter plates and shake flasks require manual interactions with each addition of feed medium. Such interactions become obsolete with the automated feeding module of the micro-Matrix. This allows for the investigation of more complex strategies that would otherwise only be feasible at the larger scale.

\section{MATERIALS \& METHODS Cell culture}

An industrial GS-CHO cell line (Lonza, UK) was expanded in a shake flask (Corning life Sciences, USA) on an orbital shaker with 25-mm orbit (Sartorius, UK) at $160 \mathrm{rpm}, 37^{\circ} \mathrm{C}$, $5 \% \mathrm{CO}_{2}$ and $70 \%$ humidity for 7 days before inoculation.

Prior to inoculation, $\mathrm{pH}$ offset values of the optical sensors within the micro-Matrix cassette (Applikon) were estimated by equilibrating the sensors in each well with PBS (Life Technologies, UK). Offline measure- 


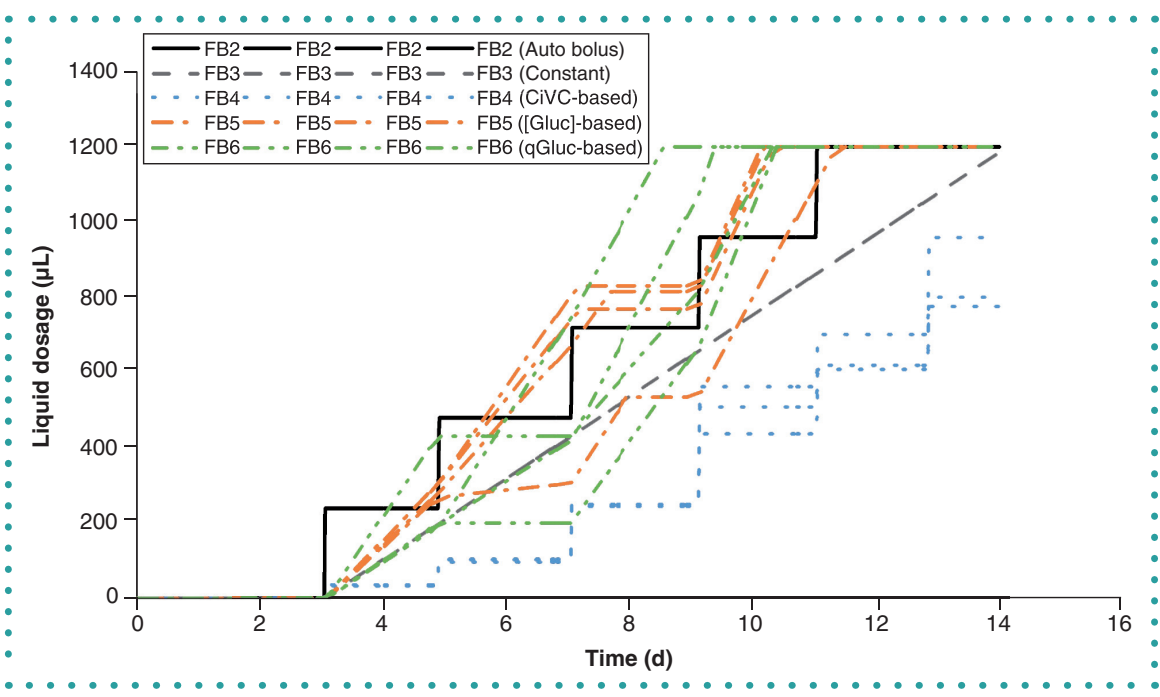

Figure 1. Accumulated additions of feed medium over the course of the cultivation as recorded by the micro-Matrix control software. Feed additions for feeding strategy FB1 were performed manually and are therefore not shown.

CiVC: Cumulative integral viable cell concentration.

$>$ ments of the same solution were then used to determine and correct the offset. Following the complete removal of PBS, each well was then inoculated with $4 \mathrm{ml}$ of cell suspension with a density of $3 \times 10^{5}$ viable cells $\mathrm{ml}^{-1}$. During the run, $\mathrm{pH}$ was controlled at 7.2 using overlay with $\mathrm{CO}_{2}$ and bolus additions of bicarbonate buffer ( $250 \mathrm{mM} \mathrm{NaHCO}_{3}$ and $250 \mathrm{mM} \mathrm{Na}_{2} \mathrm{CO}_{3}$ ); dissolved oxygen was controlled two-sided at $30 \%$ via overlay with $\mathrm{N}_{2}$ and $\mathrm{O}_{2}$. The shaking speed was set to $250 \mathrm{rpm}$. $350 \mu$ l of sample was taken from each well at least every third day. Feeding with Efficient Feed B (Life Technologies) commenced on day 3 and was set to a total volume of $1200 \mu$ l over the course of the cultivation. Six different feeding strategies were tested, as outlined below.

\section{FB1 Bolus feed addition}

The total volume of $1200 \mu$ l feed was divided into five equal additions of $240 \mu$ l over the course of the cultivation. Following day 3 , $240 \mu$ of Feed B was added manually to each well every 2 days until day 11 .

\section{FB2 Bolus feed addition automated}

Following day $3,240 \mu$ l of Feed B was added to each well every 2 days until day 11 . This was done automatically through the micro-Matrix software and required the prior calibration of the feeding module with the Feed B medium.

\section{FB3 Constant flow rate}

Following day 3 , constant feeding was initiated via the micro-Matrix software at a flow rate of
Where $V_{\text {FeedB }}$ is the new cut-off value for Feed $B$ to be added, $V_{\max }$ is the total volume of Feed $B$ to be added throughout the culture $(1200 \mu \mathrm{l})$, CiVC $_{\max }$ is the maximum expected CiVC at the end of the culture $\left(12 \times 10^{7}\right.$ cell d ml$\left.^{-1}\right), \operatorname{CiVC}_{i}$ is the current value derived from the cell count and $\operatorname{CiVC}_{\mathrm{i}-1}$ is the value derived from the previous cell count. This amount of feed medium was then added as a bolus addition to each well.

\section{FB5 Feeding based on the concentration of glucose}

On each sample day, the glucose concentration was determined. This value was then used to calculate the amount of Feed B that needs to be added to increase the concentration of glucose in each well to $6 \mathrm{~g} \mathrm{l}^{-1}$. This amount was then added using a continuous flow over the course of 2 days.

\section{FB6 Feeding based on the}

\section{consumption rate of glucose}

The glucose measurements were used to calculate the rate of glucose consumption according to Equation 3. The continuous feed addition was then adjusted to match the consumption rate of glucose.

$r_{S}=\frac{[\text { Gluc }]_{i}-[\text { Gluc }]_{i-1}}{t_{i}-t_{i-1}}$

(Equation 3) pecific glucose consumption remains constant. From previous experiments, a final CivC of $12 \times 10^{7}$ cell $\mathrm{d} \mathrm{ml}^{-1}$ was assumed Following each cell count, the CiVC was estimated according to the trapezoid method outlined in Equation 1

$\operatorname{civc}=\sum \frac{x_{i}+x_{i-1}}{2} \cdot\left(t_{i}-t_{i-1}\right)$

(Equation 1)

Where $x_{\mathrm{i}}$ is the viable cell concentration at time $t_{\mathrm{i}}$ and $x_{\mathrm{i}-1}$ the viable cell concentration at time $t_{\mathrm{i}-1}$. The trapezoid method is expected to result in a slight overestimation of the CiVC compared with alternative methods. However, as the estimated final CiVC is calculated in the same way, this inaccuracy can be neglected. The volume was then calculated according to Equation 2.

$$
V_{\text {FeedB }}=\frac{V_{\text {max }}}{\operatorname{CiVC}_{\max }} \cdot\left(C / V C_{i}-C / V C_{i-1}\right)
$$

(Equation 2)
Where [G/uc] is the concentration of glucose at time $t_{\mathrm{i}}$ and $[\mathrm{G} / \mathrm{uc}]_{\mathrm{i}-1}$ is the concentration of glucose at time $t_{\mathrm{i}-1}$.

\section{Analytics}

Samples were analyzed for viable cell concentrations using aVi-CELL XR (Beckman Coulter, UK). Before further analysis, the cells were pelleted at $1000 \times \mathrm{g}$ for $5 \mathrm{~min}$. The supernatant was then analyzed for concentrations of nutrients and metabolites using a Bioprofile Flex (Nova Biomedical, USA). The titer of IgG4 was determined through high-performance liquid chromatography using an Agilent 1200 (Agilent Technologies, UK) in combination with a 1-ml HiTrap Protein G HP column (GE Healthcare, UK).

\section{Statistics}

Statistical significance was determined using the two-tailed Student's t-test. Differences yielding a p-value below 0.05 were considered significant. 


\section{RESULTS \& DISCUSSION}

Figure 1 shows the feed added to each well over the course of the cultivation by the microMatrix system. As the bolus feeding of the first column was done manually, it is not registered by the system and therefore, not represented in the diagram. The selected feeding regimens cover a broad range of rate and time points of the feed additions. In particular, the strategies linked to the glucose concentration (FB5) and consumption rate (FB6) promote a more rapid addition in the early stages of the cultivation, whereas the CiVC-based strategy (FB4) fails to reach the total feed volume of $1200 \mu$ l even after 14 days of cultivation. With exception of the CiVC-based feeding strategy, all wells received the same amount of feed medium in total.

As shown in Figure 2, the viable cell concentration is only minimally affected by the selected feeding regimens. Most feeding strategies promote growth to cell densities of up to $1.9 \times 10^{7}$ cells $\mathrm{ml}^{-1}$, which is roughly five-times higher than the maximum cell densities achieved in typical batch process with this cell line and cultivation format [6]. Only FB4 appears to limit the growth to a maximum viable cell density of $1.15 \times 10^{7}$ cells $\mathrm{ml}^{-1}$, which is significantly lower than the maximum cell concentration of FB1 $(p=0.017)$. The final titers range between $0.98 \mathrm{~g} \mathrm{l}^{-1}$ for FB4 feeding and $1.13 \mathrm{~g} \mathrm{l}^{-1}$ for FB3. However, the difference between the highest and the lowest final titer is not signif- icant $(p=0.38)$. The viability was maintained above $70 \%$ for all feeding strategies over the duration of the experiment.

As depicted in Figure 3, the general trend of the glucose concentration for each feeding strategy is similar over the course of the cultivation. FB4 reaches glucose depletion first, after 6 days, and the glucose level only increases again towards the end of the cultivation. Due to the comparatively low additions of feed medium in this condition, the cells are starved of glucose, which is also reflected in the lower viable cell concentration. FB5 and FB6 are particularly successful in maintaining glucose levels of $1 \mathrm{gl}^{-1}$ and above for the majority of the cultivation. As FB6 was intended to match the glucose addition to the glucose consumption, a decreasing glucose concentration was unexpected. However, feeding was based on the glucose consumption of the preceding time interval and therefore likely underestimated the actual glucose requirements during the growth phase. The lactate profile shows a steady increase until day 7 , followed by lactate consumption and eventually depletion of lactate in most conditions. In case of FB4, the lactate shift occurs earlier at around day 5 of the cultivation. The literature suggests that this could be caused by the limited availability of glucose $[23,24]$. The consumption of lactate has frequently been reported to coincide with an increase in productivity [24], which could explain the compa- rable final titer of FB4. The consumption of glutamate progresses similarly in all conditions. Glutamate depletion is reached around day 7 of cultivation for FB1, FB2 and FB4, and around day 9 of cultivation for the remaining feeding strategies.

The results show that the chosen feeding strategies only have a limited effect on the growth and production kinetics of the GS-CHO cell line used; within a certain range of glucose concentrations the cell line appears to behave very robustly. Growth is only restricted when glucose becomes severely limited, as is the case for FB4. However, the final titer was comparable to the other feeding strategies, which indicates an increased specific productivity for FB4. The effect of a changing feeding strategy is highly dependent on the cell line and its sensitivity. Unchanged growth and production profiles, even for very different feeding regimens, are not out of the ordinary [20].

More complex feeding strategies based on nutrient or cell concentrations are time consuming to execute and require larger sample volumes. As these feeding strategies did not present an advantage in this study, selecting a simpler feeding regimen such as bolus or continuous addition at a constant rate could be justified. By using the feeding module to automate the feed additions, manual interactions with the system can be decreased substantially, which in turn decreases the risk of contamination and human error.
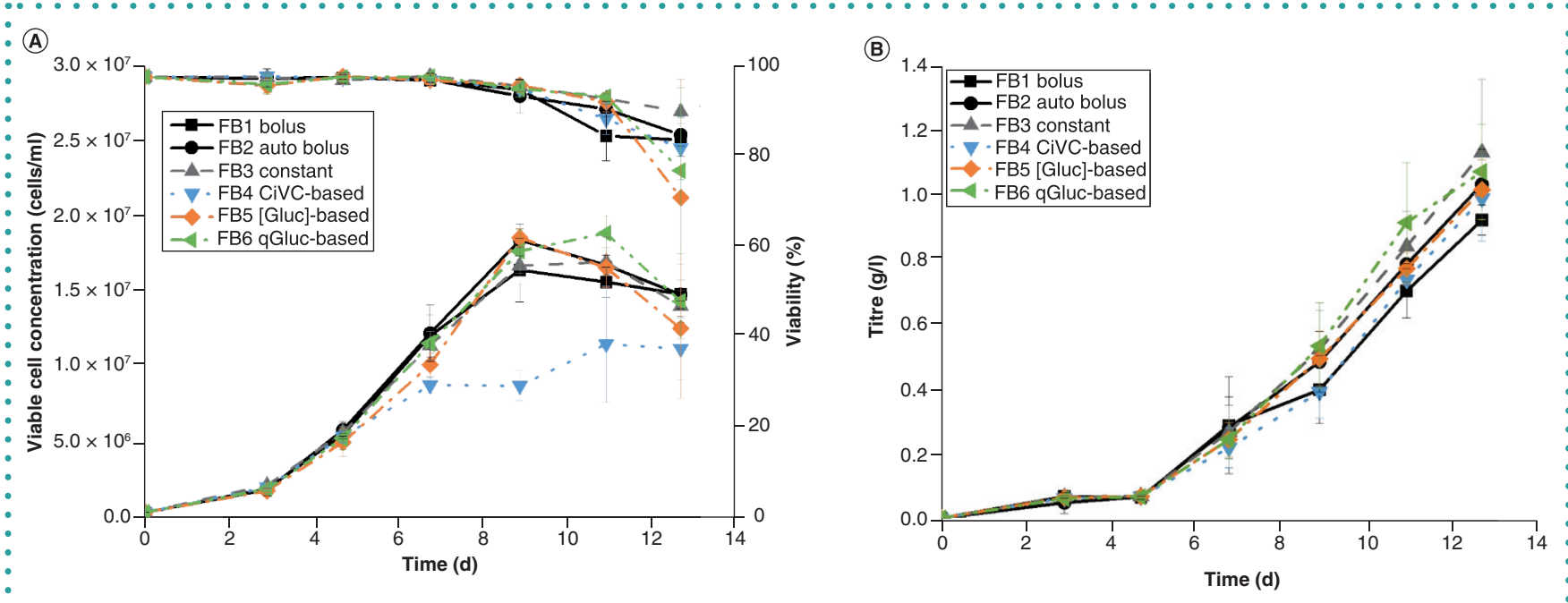

Figure 2. Growth and viability (A) and IgG4 production (B) profiles of a GS-CHO cell line grown with various feeding strategies in the micro-Matrix bioreactor at a shaking speed of $250 \mathrm{rpm}$, a working volume of $4 \mathrm{ml}$ and active control of temperature $\left(37^{\circ} \mathrm{C}\right), \mathrm{pH}(7.2)$ and dissolved oxygen $(30 \%)$. The error bars represent one standard deviation about the mean $(n \geq 3)$.

CivC: Cumulative integral viable cell concentration. 

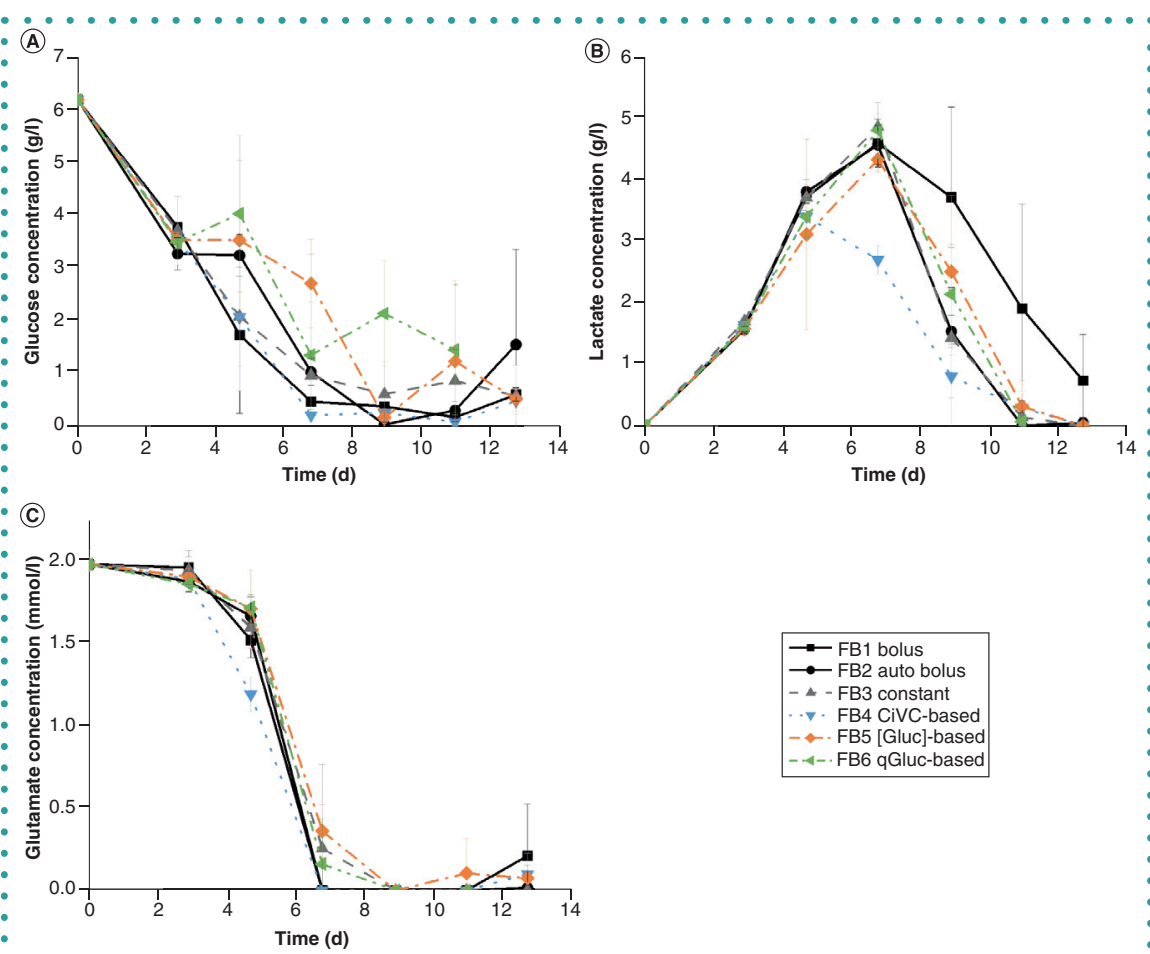

Figure 3. Glucose (A), lactate (B) and glutamate (C) profiles of a GS-CHO cell line grown with various feeding strategies in the micro-Matrix bioreactor at a shaking speed of $250 \mathrm{rpm}$, a working volume of $4 \mathrm{ml}$ and active control of temperature $\left(37^{\circ} \mathrm{C}\right), \mathrm{pH}(7.2)$ and dissolved oxygen (30\%). The error bars represent one standard deviation about the mean $(n \geq 3)$.

Civc: Cumulative integral viable cell concentration.

\section{- FUTURE PERSPECTIVE}

The automation of feed additions at small scale potentially allows for the rapid development of full-scale, fed-batch processes. To take full advantage of such systems, statistical tools such as DoE are ideal and have a strong record of accomplishment. Future experiments will therefore employ model-assisted DoE with the aim to create predictive models for the optimization of the fed-batch process. In this context, more glucose-restricted strategies will also be considered, as the present data imply an increased specific productivity for lower overall feed volumes. Furthermore, the scalability of results generated when using the micro-Matrix system to stirred benchtop bioreactors will be investigated.

\section{AUTHOR CONTRIBUTIONS}

VW: Planning and execution of experiments and writing of the paper; MG: Assistance in planning and executing experiments; $\mathrm{CBM}$ : Supervision and proofreading; FB: Experimental design, supervision and proofreading.

\section{ACKNOWLEDGMENTS}

The authors would like to thank Lonza for provision of the GS-CHO cell line.

\section{FINANCIAL \& COMPETING INTERESTS DISCLOSURE}

This work was supported by the UK Engineering and Physical Sciences Research Council (EPSRC) Industrial Doctorate Training Centre (IDTC) in Bioprocess Engineering leadership (EP/G034656/1). VW would also like to thank Applikon Biotechnology for additional financial support of his EngD studentship. The authors have no other relevant affiliations or financial involvement with any organization or entity with a financial interest in or financial conflict with the subject matter or materials discussed in the manuscript apart from those disclosed.

No writing assistance was utilized in the production of this manuscript.

\section{OPEN ACCESS}

This work is licensed under the AttributionNonCommercial-NoDerivatives 4.0 Unported License. To view a copy of this license, visit http://creativecommons.org/licenses/by-nc$\mathrm{nd} / 4.0 /$

\section{REFERENCES}

1. Hemmerich J, Noack S, Wiechert W, Oldiges M. Microbioreactor systems for accelerated bioprocess development. Biotechnol. J. 13(4), 1700141 (2018)

2. Kim BJ, Diao J, Shuler ML. Mini-scale bioprocessing systems for highly parallel animal cell cultures. Biotechnol. Prog. 28(3), 595-607 (2012).

3. Amanullah A, Otero JM, Mikola $\mathrm{M}$ et al. Novel micro-bioreactor high throughput technology for cell culture process development: Reproducibility and scalability assessment of fed-batch CHO cultures. Biotechnol. Bioeng. 106(1), 57-67 (2010)

4. Betts JPJ, Warr SRC, Finka GB et al. Impact of aeration 24-well miniature bioreactor. Biochem. Eng. J. 82, 105-116 (2014).

5. Hsu W-T, Aulakh RPS, Traul DL, Yuk IH. Advanced microscale bioreactor system: a representative scale-down model for bench-top bioreactors. Cytotechnology 64(6), 667-678 (2012).

6. Wiegmann V, Martinez CB, Baganz F. A simple method to determine evaporation and compensate for liquid losses in small-scale cell culture systems. Biotechnol. Lett. 40(7), 1029-1036 (2018).

7. Rodrigues ME, Costa AR, Henriques M, Azeredo J, Oliveira R. Technological progresses in monoclonal antibody production systems. Biotechnol. Prog. 26(2), 332-351 (2010).

8. Vallée $C$, Durocher $Y$, Henry 0 . Exploiting the metabolism of PYC expressing HEK293 cells in fed-batch cultures. $J$. Biotechnol. 169, 63-70 (2014).

9. Wlaschin KF, Hu W-S. Fedbatch Culture and Dynamic Nutrient Feeding. Springer, Berlin, Heidelberg, Germany, 43-74 (2006).

10. Lim HC, Shin HS. Fed-Batch Cultures: Principles and Applications of Semi-Batch Bioreactors. Cambridge University Press, Cambridge, UK (2013).

11. Bibila TA, Robinson DK. In pursuit of the optimal fed-batch process for monoclonal antibody production. Biotechnol. Prog. 11(1), 1-13 (1995).

12. Jo EC, Park HJ, Park JM, Kim KH. Balanced nutrient fortification enables high-density hybridoma cell culture in batch culture. Biotechnol. Bioeng. 36(7), 717-722 (1990).

13. Luan YT, Mutharasan R, Magee WE. Strategies to extend longevity of hybridomas in culture and promote yield of monoclonal antibodies. Biotechnol. Lett. 9(10), 691-696 (1987).

14. Jo EC, Park HJ, Kim DI, Moon HM. Repeated fed-batch culture of hybridoma cells in nutrient-fortified high-density medium. Biotechnol. Bioeng. 42(10), 1229-1237 (1993).

15. Li F, Vijayasankaran N, Shen AY, Kiss R, Amanullah A. Cell culture processes for monoclonal antibody production. MAbs. 2(5), 466-479 (2010).

16. Silk NJ, Denby S, Lewis $\mathrm{G}$ et al. Fed-batch operation of an industrial cell culture process in shaken microwells. Biotechnol. Lett. 32(1), 73-78 (2010).

17. Xie L, Nyberg G, Gu X et al. Gamma-interferon production and quality in stoichiometric fed-batch cultures of Chinese hamster ovary (CHO) cells under serum-free conditions. Biotechnol. Bioeng. 56(5), 577-582 (1997).

18. Zhou W, Rehm J, Hu W-S. High viable cell concentration fed-batch cultures of hybridoma cells through on-line nutrient feeding. Biotechnol. Bioeng. 46(6), 579-587 (1995).

19. Chee Furng Wong D, Tin Kam Wong K, Tang Goh L, Kiat Heng C, Gek Sim Yap M. Impact of dynamic online fed-batch strategies on metabolism, productivity and $\mathrm{N}$-glycosylation quality in $\mathrm{CHO}$ cell cultures. Biotechnol. Bioeng. 89(2), 164-177 (2005).

20. Lu F, Toh PC, Burnett I et al. Automated dynamic fed-batch process and media optimization for high productivity cell culture process development. Biotechnol. Bioeng. 110(1), 191-205 (2013).

21. Möller J, Kuchemüller KB, Steinmetz T, Koopmann KS Pörtner R. Model-assisted design of experiments as a concept for knowledge-based bioprocess development. Bioprocess Biosyst. Eng. 42(5), 867-882 (2019).

22. Kuwae S, Ohda T, Tamashima H, Miki H, Kobayashi K. Development of a fed-batch culture process for enhanced production of recombinant human antithrombin by Chinese hamster ovary cells. J. Biosci. Bioeng. 100(5), 502-510 (2005).

23. Altamirano C, Paredes C, Illanes A, Cairó J, Gòdia F. Strategies for fed-batch cultivation of t-PA producing $\mathrm{CHO}$ cells: substitution of glucose and glutamine and rational design of culture medium. J. Biotechnol. 110(2), 171-179 (2004).

24. Altamirano $C$, Illanes $A$, Becerra S, Cairó JJ, Gòdia F. Considerations on the lactate consumption by $\mathrm{CHO}$ cells in the presence of galactose. J. Biotechnol. 125(4), 547-556 (2006). 